\title{
Diagnostic Accuracy of Conventional Cell Blocks Along with p16INK4 and Ki67 Biomarkers as Triage Tests in Resource-poor Organized Cervical Cancer Screening Programs
}

\author{
Fanny Desai ${ }^{1}$, Lisam Shanjukumar Singh ${ }^{1 *}$, Gracy Majachunglu ${ }^{2}$, Helen Kamei ${ }^{3}$
}

\begin{abstract}
Background: Liquid based cytology with dual biomarkers has improved sensitivity and specificity in detecting high grade cervical intraepithelial neoplasia (CIN). In low resource settings, especially in organized camps, LBC is costly and immunohistochemistry on conventional pap smears is difficult to standardize with consumption of lots of reagents. In present study, to improve the accuracy of conventional pap smears and reduce the cost of biomarker testing, we evaluated conventional cell blocks (CCBs) preparations with biomarkers to detect high-grade CIN in resource-poor organized screening programs. We also studied feasibility of using CCB as primary screening test. Material and Methods: A total of 350 participants were included in the cross-sectional evaluation of the screening tests. A conventional Papanicolaou (Pap) smear was obtained, and another sample was then collected and placed in $10 \%$ neutral buffered formalin for CB preparation. All abnormal Pap tests and CBs were stained for the biomarkers p16INK4a and Ki67. Histopathology with p16INK4a expression was considered the gold standard. Diagnostic tests were compared using MacNemar's test and receiver operating curves were plotted. Results: The sensitivity, specificity, and diagnostic accuracy of CCB cytology, $\mathrm{CB}+\mathrm{p} 16$ cytology and $\mathrm{CB}+\mathrm{p} 16 \mathrm{Ki} 67$ cytology for detecting CIN2+ lesions were $85.71 \%, 100 \%, 97.44 \%$; $100 \%, 93.75 \%, 94.87 \%$; and $85.71 \%, 100 \%, 97.44 \%$, respectively. The Ki67 index could further categorize low grade lesions into lesions with low proliferative index and with high proliferative index (Pearson chi-square $p$ value $<0.001$ ). Conclusion: If CB preparation is standardized, CCB cytology with biomarkers can have better diagnostic accuracy than conventional cytology, can classify low grade lesions likely to progress and can be used in field settings as primary screening test.
\end{abstract}

Keywords: Diagnostic accuracy- Conventional Cell block- p16INK4a- Ki67- cervical cancer screening

Asian Pac J Cancer Prev, 20 (3), 917-923

\section{Introduction}

The main aim of cervical cancer screening is to detect and treat high-grade cervical intraepithelial neoplasia (CIN) to prevent its progression into invasive cancer; hence, a screening test should have optimal sensitivity and specificity for detecting these lesions (Arbyn et al., 2009). Commonly used Papanicolaou (Pap) screening tests could not reduce death rates due to cervical cancer in the developing world (Ferlay et al., 2012) due to many factors. One of the reasons is lower accuracy of pap smears. Pap smears have variable sensitivity, poor reproducibility, inter- and intraobserver variations and require repeated visits in some cases, which is more cumbersome in low-resource settings (Catarino et al., 2015; Ogilvie et al., 2017). Although there are a few advantages of liquid-based cytology, conventional and liquid-based cytology have similar sensitivity and specificity (Arbyn et al., 2008). The sensitivity and specificity of conventional and liquid-based cytology tests have been reported to be $26-70 \%$ and $96-99 \%$, respectively (Arbyn et al., 2008; Bergeron et al., 2015). Other methods have been recommended recently, such as HPV (human papilloma virus) testing and the VIA (visual inspection with acetic acid) method, but they have their limitations. HPV testing requires a follow-up triage test, as the majority of HPV infections regress and do not advance to invasive cervical cancers (Rajaraman et al., 2015). The VIA method has reduced cancer deaths, but it is operator-dependent, with an increased risk of unnecessary treatments in women of reproductive age, and has been reported to have reduced sensitivity and specificity in postmenopausal women (Li et al., 2009; Sankaranarayanan et al., 2007). Cytology with dual biomarkers has shown improved sensitivity and specificity. These studies used liquid 
based cytology (Ikenberg et al., 2013; Carozzi et al., 2008) and cell blocks made from residual material (Liu et al., 2007; Ngugi et al., 2015). In resource poor settings, where screening is conducted mainly by outreach camps, liquid based cytology is costly and biomarker testing on conventional pap smears will consume lots of reagents. To improve the accuracy of conventional pap smears and reduce the cost at the same time, we used biomarkers p16INK4a and Ki67 on conventional cell blocks (CCB: Cell Block prepared from samples collected by conventional technique). We also studied feasibility of using $\mathrm{CCB}$ as a primary screening test to detect high-grade $\mathrm{CIN}$ in resource-poor organized screening programs, so that the total number of preparations and their examination can be reduced. We also discuss different gel-based CB preparation methods and their diagnostic yield and utility in the field setting and also describe the novel filtration based approach.

\section{Materials and Methods}

After Institutional Ethical Committee approval, we conducted cervical cancer screening camps in different parts of Manipur between the period of August 2015 and January 2016. An awareness program was conducted, and the local health providers invited the participants to the each camp. Indigenous Manipuri females between 28 and 70 years of age who gave informed consent, included in the study. Females above 65 years of age with three previous negative Pap smears, unmarried females and female less than 28 years of age were excluded from the group. The cross-sectional analysis evaluated the efficacy of the conventional Pap test, CCB test, CCB with p16INK4a cytology and CCB with p16INK4a + Ki67 index.

\section{Sample collection and processing}

First, conventional Pap smears were obtained using sterile wooden spatulas and endocervical brushes and material was placed on three glass slides, which were immediately fixed in $80 \%$ isopropyl alcohol. The ends of both sample collectors were then cut and placed in 10\% neutral buffered formalin. Another sample taken with a wooden spatula was placed into the same fixative vial without dispersion. All samples were collected by a trained local health provider. The samples were then transported to the lab and processed the following day. Pap smears were stained with Pap stain. CBs were prepared from the formalin-fixed samples. The fixation time was 6-12 hr.

\section{Cell block preparation}

First, all tissue particles attached to the spatula and endocervical brush were dislodged by toothless forceps into the fixative. Then, the samples were triaged according to particle size. Samples with abundant large visible particles were filtered with a porous filter and placed into cassettes. Samples with small particles were filtered with a fine filter in a filtration unit, the pellet was wrapped in filter paper and processed as a conventional sample. Samples with scanty material were centrifuged at 2,000 RPM for $10 \mathrm{~min}$. For the gel-conical tube method, the supernatant was discarded, and an equal volume of melted $2 \%$ agar gel $\left(50^{\circ} \mathrm{C}\right)$ was added. The sediment was resuspended, recentrifuged at 2,000 RPM for $5 \mathrm{~min}$ and then incubated at $2-8^{\circ} \mathrm{C}$ for $30 \mathrm{~min}$ (the average preparation time was 45-50 min). The gel block was dislodged with a long blunt stick, cut in half, placed onto a piece of filter paper, and immersed in formalin for $6 \mathrm{hr}$ before processing. For the flat-bottom tube method (Figure 1D), we transferred the sediments into a flat-bottom tube and prepared the CB according to Shiddham's protocol (Varsegi and Sidham, 2009). In the third method (fine filtration + cell-gel method) for visibly low cellular samples, the sediment was transferred into a filtration unit for 2-3 $\mathrm{min}$ and the pellet was retrieved. Then, eosin drop and $2 \%$ partially melted agar gel were added. After gel solidification, the pellet was wrapped in filter paper and processed as a routine histopathology specimen. No repeated centrifugation steps were required in the latter method. The average preparation time was $15 \mathrm{~min}$. Tissues were submitted to routine tissue processing. Tissue cassettes were removed from wax and embedded, and sections were cut with a thickness of $4 \mu \mathrm{m}$. Sections with maximum eosin staining were selected (eosin was added to low cellular samples at the time of cell pellet preparation). The sections were stained with hematoxylin and eosin (H and $\mathrm{E})$.

\section{Examination and reporting}

Both CCBs and Pap smears were analyzed according to the Bethesda 2014 system (Nayar and Wilbur, 2015) by two experts blinded to both results. Squamous cell lesions were classified as NILM (negative for intraepithelial lesion or malignancy) (Figure 1A and 1B (Atrophy), ASCUS (atypical squamous cells of undetermined significance), LSIL (low-grade squamous intraepithelial lesion) (Figure 1C), ASC-H (atypical squamous cell, high-grade lesion cannot be ruled out), HSIL (high-grade squamous intraepithelial lesion), squamous cell carcinoma in situ (Figure 1D), and squamous cell carcinoma (Figure 1E). Glandular lesions were classified as benign glandular cells, atypical glandular cells-NOS type, atypical glandular cells-favors neoplastic, adenocarcinoma in situ and adenocarcinoma (Figure 1F). Immunohistochemistry was performed on cases that were abnormal on Pap and CB cytology. Several cases were randomly assigned as controls. A specimen was considered adequate when $>100$ cells of interest were found and was considered partially adequate when there were 50-100 cells of interest. The specimen was reported inadequate when cells of interest were less than 50 cells or only mucous, blood, or inflammation was present. Each specimen was evaluated for adequacy according to the age of the patient. The transformation zone and presence of endocervical cells were noted.

\section{Immunohistochemical (IHC) stain protocol}

Immunohistochemisty was carried out on 4 micron thick sections using anti-p16INK4a (Biogenix, Fremont, USA, clone G175-405) and anti Ki67 (Dako, Denmark, clone MIB 1) as primary antibodies and Dako envision system as detection kit. IHC was carried out manually 
using manufacturer's standardized protocol. We stained a positive and negative control for each batch. Cervical cancer was used as the positive control, and the primary antibody was omitted as the negative control.

\section{Biomarker reporting}

The expression of the biomarker p16INK4a was reported as negative, weakly positive and strongly positive (Figure 1G) for each block. The Ki67 labeling index was measured using image analysis. We used NIH image J- cell counter plugin for counting KI67 positive cells. Hotspot areas with 100-200 cells were counted and reported as percentages. In small fragments where 100 cells were not found, similar morphology fragments were identified on $\mathrm{CB}$ sections, and mitosis was counted. The Ki67 expression pattern in CBs was described as basal, middle layer, superficial-discohesive (Figure 1H), fragment-like (Figure 1I) and inconclusive. Spectrum of different CB lesions with their respective triage tests expressions has been uploaded as supplementary file-1 Dual positivity ( 1 16INK4a + Ki67) in cells of interest was considered as sign of neoplastic transformation and final diagnosis of HSIL was given only when CB morphology suggested full thickness epithelial involvement.

\section{Colposcopy and treatment referral}

Based on the screening results of the Pap test only, participants were advised on colposcopic-guided biopsies and treatment according to the World Health Organization (WHO) guidelines (Broutet et al., 2013). Participants with normal Pap and abnormal CB cytology results were kept under follow-up.

\section{Cervical biopsies}

All cases with abnormal Pap tests underwent colposcopy referral, and multiple punch biopsies were taken in indicated cases. p16INK4a staining was performed on all biopsies, and histopathology + p16INK4a (Figure 1J) was considered the gold standard according to recent WHO recommendations (Khurman et al., 2014). Although two-tiered classification is recommended, we classified precursor lesions as CIN-1, CIN2 (HSIL) and CIN3 (HSIL) on biopsies for comparison with previous studies.

\section{Statistical analysis}

Statistical analysis was performed using IBM SPSS Statistics 21. Pearson's chi-square test was used to measure the relationships and associations between the variables. Cohen's kappa agreement was calculated to assess concordance between different diagnostic tests and gold standard. MacNemar test used to compare Pap test and $\mathrm{CB}$ test with combination of triage tests. ROC curves were plotted, and sensitivity, specificity, positive predictive value, negative predictive value and diagnostic accuracy were calculated. Alpha values $<0.05$ were considered statistically significant. Verification bias was not analyzed as it would be impractical to do biopsies in unindicated cases.

\section{Results}

A total of 350 participants $196(56 \%)$ from hilly region and 154 (44\%) from valley regions were included in this cross-sectional evaluation of the screening tests. Between age group 28-30 there were 34 participants, between $31-40,121 ; 41-50,105 ; 51-60,64$ and 61-70, 26. The procedures were well tolerated in 339 (96.86\%) cases. CBs were prepared for 325 cases. Pap smears showed adequate cellularity in $346(98.9 \%)$ cases. CB cellularity was adequate in 286 cases $(88 \%)$, inadequate

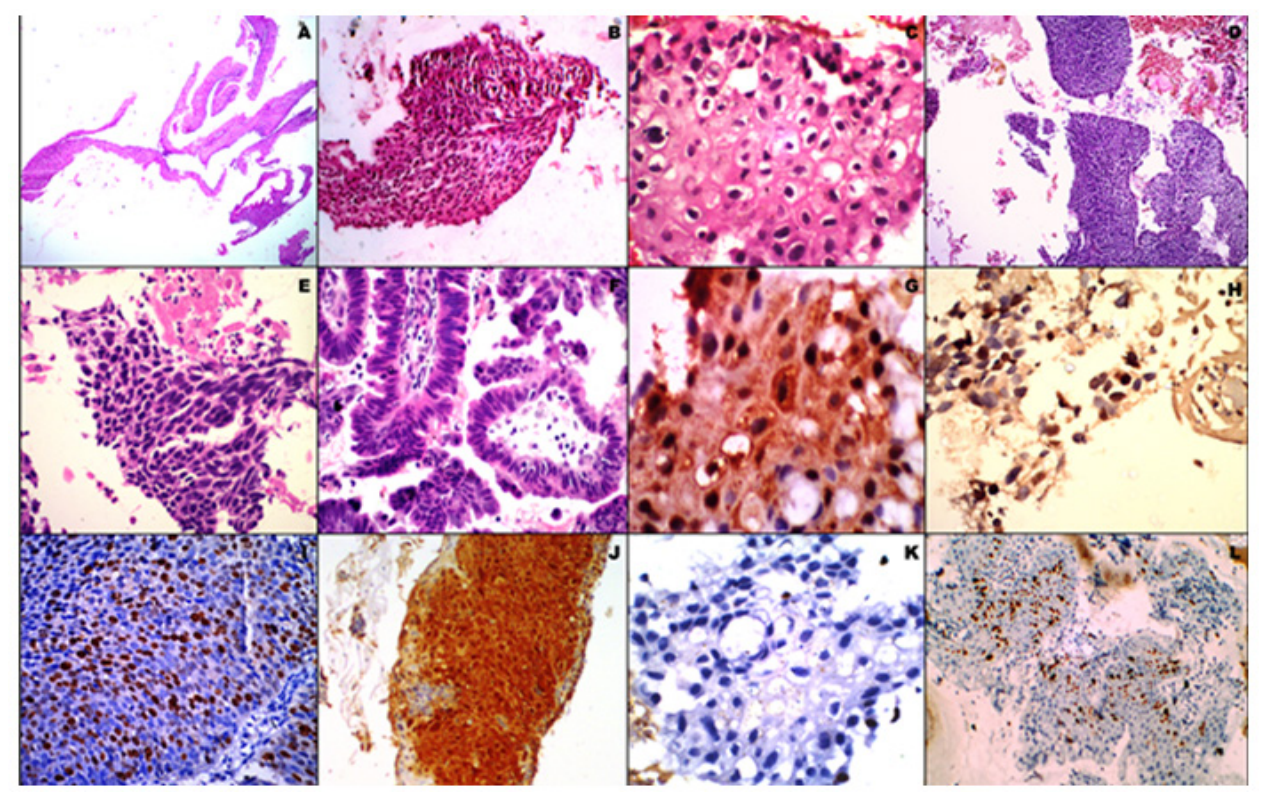

Figure 1. Spectrum of Lesions on Cell Blocks. (A) NILM, H\&E, 5 x (CB); (B) Atrophy, H\&E, 10x (CB); (C) LSIL, H\&E, 40x(CB); (D) Carcinoma in situ, H\&E, 10x (CB); (E) Squamous cell carcinoma, H\&E, 40x (CB); (F) Adenocarcinoma, H\&E, 40x (CB); (G) LSIL with low Ki67, p16INK4, 40x; (H) HSIL, Ki67, 10x (CB) (superficial and discohesive pattern); (I) Carcinoma in situ, Ki67, 40x (CB) (fragment-like pattern); (J) HSIL (CIN3), p16INK4a, 10x (biopsy); (K) LSIL with low Ki67 index, 40x; (L) LSIL with high Ki67 index, 10x. 
Table 1. Pap test, CB test, Triage Tests and Gold Standard Correlations

\begin{tabular}{|c|c|c|c|c|c|c|}
\hline \multirow[t]{2}{*}{ Diagnostic test } & \multicolumn{5}{|c|}{ Gold standard } & \multirow[t]{2}{*}{ Total } \\
\hline & NILM & CIN1 & CIN 2 & CIN 3 & CANCER & \\
\hline \multicolumn{7}{|l|}{ Pap diagnosis } \\
\hline NILM & 4 & 1 & 0 & 0 & 0 & 5 \\
\hline ASCUS & 5 & 5 & 1 & 0 & 0 & 11 \\
\hline LSIL & 4 & 1 & 0 & 0 & 0 & 5 \\
\hline ASC-H & 7 & 2 & 1 & 0 & 0 & 10 \\
\hline HSIL & 1 & 2 & 1 & 1 & 2 & 7 \\
\hline Squamous cell carcinoma & 0 & 0 & 0 & 0 & 1 & 1 \\
\hline Total & 21 & 11 & 3 & 1 & 3 & 39 \\
\hline \multicolumn{7}{|l|}{ CB diagnosis } \\
\hline ASCUS & 1 & 4 & 0 & 0 & 0 & 5 \\
\hline NILM & 9 & 1 & 0 & 0 & 0 & 10 \\
\hline LSIL & 1 & 3 & 1 & 0 & 0 & 5 \\
\hline ASC-H & 2 & 3 & 0 & 0 & 0 & 5 \\
\hline HSIL & 0 & 0 & 2 & 1 & 1 & 4 \\
\hline Atrophy & 8 & 0 & 0 & 0 & 0 & 8 \\
\hline Squamous cell carcinoma & 0 & 0 & 0 & 0 & 1 & 1 \\
\hline Adenocarcinoma & 0 & 0 & 0 & 0 & 1 & 1 \\
\hline Total & 21 & 11 & 3 & 1 & 3 & 39 \\
\hline \multicolumn{7}{|l|}{ P16INK4a cytology } \\
\hline Weak & 6 & 4 & 0 & 0 & 0 & 10 \\
\hline Strong & 0 & 1 & 2 & 1 & 2 & 6 \\
\hline Weak and strong & 0 & 1 & 1 & 0 & 1 & 3 \\
\hline Negative & 15 & 5 & 0 & 0 & 0 & 20 \\
\hline Total & 21 & 11 & 3 & 1 & 3 & 39 \\
\hline \multicolumn{7}{|c|}{$\mathrm{CB}+\mathrm{p} 16 \mathrm{INK} 4 \mathrm{a}+\mathrm{Ki67}$ Cytology } \\
\hline NILM & 17 & 5 & 0 & 0 & 0 & 22 \\
\hline LSIL & 4 & 6 & 1 & 0 & 0 & 11 \\
\hline HSIL & 0 & 0 & 2 & 1 & 1 & 4 \\
\hline Cancer & 0 & 0 & 0 & 0 & 2 & 2 \\
\hline Total & 21 & 11 & 3 & 1 & 3 & 39 \\
\hline
\end{tabular}

NILM, Negative for intraepithelial lesion or malignancy; ASCUS, Atypical squamous cell of undetermined significance; ASCH, Atypical squamous cell high-grade lesion cannot be ruled out. LSIL, Low-grade squamous intraepithelial lesion; HSIL, High-grade squamous intraepithelial lesion; SIL, Squamous intraepithelial lesion. Pap test, Papanicolaou test; CB test, Cell block test; CIN, Cervical intraepithelial lesion.

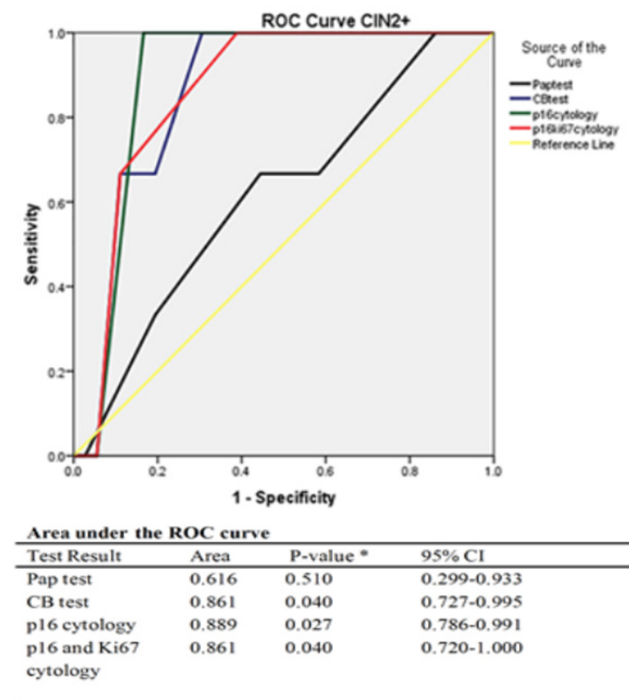

Figure 2. Receiver Operating Curve for CIN2+ Lesions with Area under the Curve (AUC) in $21(6.5 \%)$ and partially adequate in $18(5.5 \%)$ cases. We prepared CBs by simple filtration for $188(57.8 \%)$ samples, fine filtration using a filtration unit for 21 (6.5\%) samples, the gel-conical method for $95(29.2 \%)$ samples, and the flat-bottom method for $21(6.5 \%)$ samples. We found heat artifacts in $16(4.9 \%)$, dispersed cells in $14(4.3 \%)$, and low cellularity in $30(9.2 \%)$ cases. Samples were adequate without artifacts in $265(81.5 \%)$ cases. Immunohistochemistry was performed in 224 (64\%) cases. p16INK4a expression on CBs was weak in $60(26.8 \%)$, strong in 17 (7.59\%), weak and strong in $20(8.9 \%)$ and negative in $127(56.7 \%)$ cases. Ki67 expression was positive in $82(36.6 \%)$, negative in 139 (62.1\%) and inconclusive in $3(1.3 \%)$ cases. CBp16KI67 cytology showed LSIL in 57 (25.4\%), HSIL in $4(1.8 \%)$, carcinoma in $2(0.9 \%)$, NILM in $142(63.4 \%)$ and inconclusive results in 19 (8.5\%) cases.

Biopsy results were available for 39 cases. The different 
DOI:10.31557/APJCP.2019.20.3.917

Diagnostic Accuracy of Conventional Cell Block Screening in Cervical Cancer

Table 2. Cross-sectional Sensitivity, Specificity, PPV, NPV and Diagnostic Accuracy of Different Tests for Squamous and Glandular Lesions

\begin{tabular}{|c|c|c|c|c|c|}
\hline Diagnostic Test with different outcomes & $\begin{array}{c}\text { Sensitivity \% } \\
(95 \% \mathrm{CI})\end{array}$ & $\begin{array}{l}\text { Specificity \% } \\
(95 \% \text { CI })\end{array}$ & $\begin{array}{c}\text { PPV \% } \\
(95 \% \mathrm{CI})\end{array}$ & $\begin{array}{c}\text { NPV \% } \\
(95 \% \mathrm{CI})\end{array}$ & $\begin{array}{c}\text { Accuracy \% } \\
(95 \% \mathrm{CI})\end{array}$ \\
\hline \multicolumn{6}{|l|}{ ASCUS+ } \\
\hline Pap test & $100(59.0-100)$ & $15.62(5.3-32.8)$ & $20.59(18.3-23.1)$ & 100 & $30.77(17.0-47.6)$ \\
\hline CB test & $100(59.0-100)$ & $56.25(37.7-73.6)$ & $33.33(25.2-42.5)$ & 100 & $64.10(47.2-78.8)$ \\
\hline \multicolumn{6}{|l|}{$\mathrm{ASCH}+$} \\
\hline Pap test & $85.71(42.1-99.6)$ & $62.50(43.7-78.9)$ & $33.33(22.6-46.2)$ & $95.24(76.2-99.2)$ & $66.67(49.8-80.9)$ \\
\hline CB test & $85.71(42.1-99.6)$ & $84.38(67.2-94.7)$ & $54.55(33.7-73.9)$ & $96.43(81.4-99.4)$ & $84.62(69.5-94.1)$ \\
\hline \multicolumn{6}{|l|}{$\mathrm{CIN} 2+$} \\
\hline Pap test & $71.43(29.0-96.3)$ & $90.62(74.9-98.0)$ & $62.50(33.9-84.4)$ & $93.55(81.7-97.9)$ & $87.18(72.6-95.7)$ \\
\hline CB test & $85.71(42.1-99.6)$ & $100(89.1-100)$ & 100 & $96.97(83.9-99.5)$ & $97.44(86.5-99.9)$ \\
\hline $\mathrm{CB}+\mathrm{p} 16 \mathrm{INK} 4 \mathrm{a}$ cytology & $100(59.0-100)$ & $93.75(79.2-99.2)$ & $77.78(47.8-93.0)$ & 100 & $94.87(82.7-99.4)$ \\
\hline $\mathrm{CB}+\mathrm{P} 16 \mathrm{INK} 4 \mathrm{a}+\mathrm{Ki} 67$ cytology & $85.71(42.1-99.6)$ & $100(89.1-100)$ & 100 & 96.97 (83.9-99.5) & $97.44(86.5-99.9)$ \\
\hline \multicolumn{6}{|l|}{$\mathrm{CIN} 3+$} \\
\hline Pap test & $25(0.6-80.6)$ & $100(90-100)$ & 100 & $92.11(86.9-95.4)$ & $92.31(79.1-98.4)$ \\
\hline CB test & $50(6.8-93.2)$ & $100(90-100)$ & 100 & 94.59 (86.8-97.9) & $94.87(82.7-99.4)$ \\
\hline \multicolumn{6}{|l|}{$\mathrm{AGC}+$} \\
\hline Pap test & $0(0-97.5)$ & $73.68(56.9-86.6)$ & 0 & 96.55 (95.9-97.1) & $71.79(55.1-85.0)$ \\
\hline CB test & $100(2.5-100)$ & $100(90.7-100)$ & 100 & 100 & $100(90.9-100)$ \\
\hline
\end{tabular}

$\mathrm{CI}$, confidence interval; AGC, atypical glandular cells.

Table 3. Comparison of Sensitivity, Specificity of Cell Block Test, p16 Cytology and p16-Ki67 Cytology with Results of Different Authors

\begin{tabular}{lcc}
\hline Test/Outcome CIN2+ & Sensitivity \% (95\%CI) & Specificity \% (95\% CI) \\
\hline Cell Block & $85.71(42.13-99.64)$ & $100(89.11-100)$ \\
Present study & $86.3 \%$ & $100 \%$ \\
Gangane N et al.,2007 & & \\
p16 alone cytology & $100(59.04-100)$ & $93.75(79.19-99.23)$ \\
Present study & $88 \%(80-94)$ & $61 \%(57-64)$ \\
Carozzi F et al., 2008 & & $100(89.11-100)$ \\
CB + p16 and Ki67 cytology & $85.71(42.13-99.64)$ & $95.2 \%(94.9-95.4)$ \\
Present study & $86.7 \%(81.1-90.9)$ & $100 \%$ \\
Ikenberg H et al., 2013 & $86 \%$ & $76.7 \%(71.1-81.8)$ \\
Korolczuk A et al., 2015 & $85.0 \%(73.4-92.9)$ & \\
Stanczuk GA et al.,2017 & &
\end{tabular}

tests and gold standard correlations are presented in Table 1. The Kappa agreements for the Pap test, CB test, p16 cytology test and CB p16 and Ki67 tests were slight $(\mathrm{K}=0.000)$, slight $(0.000)$, poor $(-0.002)$ and moderate (0.410), respectively. The cross-sectional sensitivity, specificity, PPV, NPV and diagnostic accuracy of the different tests with different cutoffs for squamous as well as glandular abnormalities are presented in Table 2 . The receiver operating curves for CIN 2+ lesion were plotted and the area under the curve for each test with $\mathrm{p}$ value is described in Figure 2. Respective MacNemar $\mathrm{p}$ values for CB ASCUS+ cytology, $\mathrm{CB}+\mathrm{p} 16$ cytology and $\mathrm{CB}+\mathrm{p} 16 \mathrm{Ki} 67$ cytology were $0.002,<0.001$ and $<0.001$. We used Pap ASCUs + test as comparator. STARD flow diagrams for Pap test, CB test, Combination of single or dual triage tests are uploaded as supplementary document file 2. Additional data can be produced upon request.

Ki67 expression showed a different pattern in squamous lesions on $\mathrm{CB}$. A basal staining pattern was observed in LSIL and NILM. The superficial-discohesive pattern was commonly observed in HSIL, and the fragment-like Ki67 pattern was seen in carcinoma and HSIL $(\mathrm{P}<0.001)$. For LSIL, Ki67 labeling indices on CB varied greatly from a minimum of $8 \%$ to a maximum of $43 \%$. The mean Ki67 labeling indices were $0-1 \%$ in benign glandular cells and $60 \%$ in adenocarcinoma.

\section{Discussion}

CCB cytology has not been used as a primary cervical cancer screening test before. This is the first study that has evaluated feasibility of CCB with biomarkers in resource poor organized screening programs. In the present study, we evaluated the cross-sectional accuracy of CCB as a primary screening test and compared it with conventional Pap tests along with the p16INK4a and Ki67 biomarkers as Asian Pacific Journal of Cancer Prevention, Vol 20 
a single or combined triage tests. We found that $\mathrm{CCB}$ with biomarkers as triage tests has better diagnostic accuracy than conventional Pap smear (MacNemar p value $<0.001$ ). Dual staining of LBC preparations (Li et al., 2007; Ngugi et al., 2015) has been found to improve its sensitivity and specificity. However, dual staining of $\mathrm{CCB}$ has not been studied before. In our study, we observed that these samples are like micro biopsies. The use of biomarkers with CBs could classify lesions into simple categories such as NILM, LSIL and HSIL. The Cohen's kappa agreement value for CBp16INK4p16 was higher $(\mathrm{k}=0.410$, moderate) than other tests and its results were similar to the gold standard results $(\mathrm{p}<0.001)$. The Ki67 index could further categorize LSIL lesions into lesions with low proliferative index (Figure 1K) and lesions with high proliferative index (Figure 1L) (Pearson's chi-square p-value $<0.001$ ); thus, we can identify lesions that are likely to progress.

Our sensitivity and specificity for CBp16KI67 cytology are similar to those reported by other authors, whereas the sensitivity and specificity for p16 cytology were greater than those reported by other authors (Table 3). This variable sensitivity and specificity of $\mathrm{p} 16$ cytology alone may be due to the presence of p16 expression in LSIL and other benign mimics; therefore, $\mathrm{p} 16$ expression alone without morphological interpretation and Ki 67 Index can lead to a false-positive diagnosis (Clark et al., 2016).

We used different gel-based methods and filtration based method for CB preparations in field settings. In our study, CB preparations prepared by the simple filtration and fine filtration + cell-gel methods gave adequate cellularity in $95.7 \%$ of cases. For visibly low cellular samples, the filtration-based method gave maximum cellularity $(90.5 \%)$. The CB cellularity and artifacts were dependent on the CB-preparation method, and artifacts were more common with other gel based methods (Pearson's chi-square value $\mathrm{p}<0.001$ ). The filtration-based method retrieved most of the cellular material without any significant loss during processing, and the morphology was preserved without any artifacts. This method was fast and reproducible, without unnecessary centrifugation steps and multiple samples could be handled at the same time. The other methods required multiple centrifugations, which were cumbersome and time consuming in field application.

We studied different lesions using both CBs and conventional Pap tests, and it was possible to follow and report the cases according to the Bethesda classification of interpretation. Although the preparation time was longer for CBs with biomarkers (Mean: 4 days) than for Pap smears (Mean:2 days), the overall screening time and reporting time were greatly reduced with better accuracy for CCBs compared with Pap smears (Range: screening time: CBs, 3-5 min; Conventional Pap smears, 15-20 $\min )$. Due to the preservation of cellular architecture in CCBs, we could identify microglandular hyperplasia, villi, adenocarcinoma, squamous cell carcinoma and atrophy accurately. Causes for misinterpretation on Pap smears were glandular hyperplasia, the presence of hyper chromatic crowded groups, the presence of villi, reactive changes due to severe inflammation and the presence of lower uterine fragments. Biomarkers helped us to identify benign mimics such as atypical squamous metaplasia, reactive atypia, nonspecific glandular hyperplasia and atrophy with inflammation in CBs. Gangane et al., (2007) and Keyhani et al., (2002) reported similar findings for CBs. In our study, all glandular lesions were more correctly identified on CCBs than on Pap smears (Pearson's chi-square p-value $<0.001$ ), same as reported by other authors (Pradhan et al., 2016; Xing et al., 2014).

Our study has some limitations. The overall sample size was small as it was a preliminary study. We could not follow some of the cases to evaluate their progression, especially the p16-positive low-grade lesions with high and low Ki67 indices, due to the limited time frame. We could not study inter-observer variations using the different methods and the cellularity of CBs. We observed that analysis of biomarkers in CBs was costly. In our setting, the combined cost was an average of $450 \mathrm{Rs}$, which is three times higher than the cost of Pap tests (average of $150 \mathrm{Rs}$ ).

Although biomarker analysis in CCBs is costlier than conventional pap test, in resource-poor settings where transportation, repeated follow-up camps, human resources and overtreatment of unindicated cases incur additional costs, biomarker screening using CBs may reduce repeated follow-up camps, overtreatment and anxiety among patients due to its improved diagnostic accuracy, and cancerous and precancerous lesions can be directly referred for further management. Its improved accuracy and potential to identify lesions likely to advance can make it easier for local health care providers to communicate and convince participants to undergo follow-up and treatment. It is also possible to use it as triage option in HPV positive participants. Moreover, in places such as Manipur, where infectious diseases are highly prevalent, $\mathrm{CB}$ samples collected directly into $10 \%$ neutral buffered formalin can be a safe technique (Moller et al., 2015) for the collection and transportation of specimens to referral lab. In short, If CB preparation is standardized; CCB cytology with biomarkers can have better diagnostic accuracy than conventional cytology, can classify low grade lesions likely to progress and can be used in field settings as primary screening test.

\section{Acknowledgments}

We express deep and sincere gratitude to the DST WOS-A life sciences committee for their continued financial and moral support.

\section{References}

Arbyn M, Bergeron C, Klinkhamer P, et al (2008). Liquid compared with conventional cervical cytology: a systematic review and meta-analysis. Obstet Gynecol, 111, 167-77.

Arbyn M, Sankaranarayanan R, Muwonge R, et al (2008). Pooled analysis of the accuracy of five cervical cancer screening tests assessed in eleven studied in Africa and India. Int $J$ Cancer, 123,153-60.

Arbyn M, Ronco G, Cuzick J, Wentzensen N, Castle PE (2009). How to evaluate emerging technologies in cervical cancer screening?. Int J Cancer, 125, 2489-96.

Bergeron C, Ronco G, Reuschenbach M, et al (2015). The 
clinical impact of using p16INK4a immunochemistry in cervical histopathology and cytology: An update of recent developments. Int J Cancer, 136, 2741-51.

Broutet N, Dangou JM, Fadhil I, et al (2013). WHO guidelines for screening and treatment for precancerous lesions for cervical cancer prevention. WHO Press, World Health Organization, Geneva.

Carozzi F, Confortini M, Dalla Palma P, et al (2008). New technologies for cervival cancer screening (NTCC) working group. Use of p16 INK4a over expression to increase the specificity of human papillomavirus testing: a nested substudy of the NTCC randomised controlled trial. Lancet Oncol, 9, 937-45

Catarino R, Petignat P, Dongui G, Vassilakos P(2015). Cervical cancer screening in developing countries at a cross road: Emerging technologies and policy choices. World $J$ Clin Oncol, 10, 281-90.

Clark JL, Dan Lu, Kalir T, Liu Y (2016). Overdiagnosis of HSIL on cervical biopsies: errors in p16 immunohistochemistry implementation. Hum Pathol, 55, 51-6.

Ferlay J, Soerjomataram I, Ervik M, et al (2012). GLOBOCAN 2012 v 1.0 cancer incidence and mortality worldwide: IARC cancer base (internet), Lyon France, http://gobocaniarc.fr.

Gangane N, Mukerji MS, Anshu, Sharma SM (2007). Utility of Microwave processed cell blocks as complement to cervico vaginal smears. Diagn Cytopathol, 35, 338-41.

Ikenberg H, Bergeron C, Schmidt D, et al (2013). Screening for cervical cancer precursors with p16/ki67 dual stained cytology: results of the PALMS. J Natl Cancer Inst, 105, 1550-7.

Khurman RJ, Carcangiu ML, Herrington CS, Young RH (2014). WHO Classification of Tumors of Female Reproductive Organs. IARC and WHO, Lyon, France, pp 169-206.

Korolczuk A, Orzel M, Wozniak S, Smolen A, Caban K (2015). P16/ki67 dual immunostaining in conventional cytology in women with positive papanicolau test. J Cytol Histol, 6, 358 .

Li N, Shi JF, Franceschi S, et al (2009). Different cervical cancer screening approaches in a Chinese multicenter study. $\mathrm{Br} J$ Cancer, 100, 532-37.

Liu H, Shi J, Wilkerson M, et al (2007). Immunohistochemical detection of p16INK4a in liquid based cytology specimens on cell blocks sections. Cancer, 111, 74-82.

Moller L, Schunadel L, Nitsche A, et al (2015). Evaluation of virus inactivation by formaldehyde to enhance bio safety of diagnostic electron microscopy. Virus, 7, 666-79.

Ngugi CW, Schmidt D, Wanyoro K, et al (2015). P16INK4a/ Ki67 dual stain cytology for cervical cancer screening in Thika district, Kenya. Infect Agent Cancer, 10, 25.

Ogilvie G, Nakisige C, Huh WK, et al (2017). Optimising secondary prevention of cervical cancer: recent advances and future challenges. Int J Gynaecol Obstet, 138, 15-9.

Pradhan D, Li Z, Ocque R, Patadji S, Zhao C (2016) Clinical significance of atypical glandular cells in pap tests: an analysis of more than 3000 cases at large academic women's centre. Cancer Cytopathol, 124, 589-95.

Rajaraman P, Anderson BO, Basu P, et al (2015). Recommendations for screening and early detection of common cancers in India. Lancet Oncol, 16, 352-61.

Ritu N, Devid C, Wilbur B (2015) The Bethesda System for Reporting Cervical Cytology: Definitions, Criteria, and Explanatory Notes. 3rd ed. Springer International Publishing, Switzerland, DOI: 10.1007/978-3-319-11074-5.

Sankaranarayanan R, Esmy PO, Rajkumar R, et al (2007). Effect of visual screening on cervical incidence and mortality in Tamil Nadu, India a cluster randomised trial. Lancet, 370, 398-406.

Stanczuk GA, Baxter GJ, Currie H, et al (2017). Defining optimal triage strategies for hrHPV screen-positive women-an evaluation of HPV 16/18 genotyping, cytology, and p16/ Ki-67 cytoimmunochemistry. Cancer Epidemiol Biomarkers Prev, 26, 1629-35.

Varsegi GM, Shidham V (2009). Cell block preparation from cytology specimen with predominance of individually scattered cells. J Vis Exp, 21, 1316.

Xing W, Hou A, Fischer A, Owens CL, Jiang Z (2014). The cellient automated cell block system is useful in differential diagnosis of atypical glandular cells in papanicolaou tests. Cancer Cytopathol, 122, 8-14.

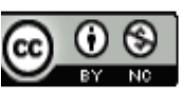

This work is licensed under a Creative Commons AttributionNon Commercial 4.0 International License. 